\title{
Peroral pancreatoscopy with electrohydraulic litho- tripsy for pancreatic duct stone after placement of fully covered self-expandable metal stent
}

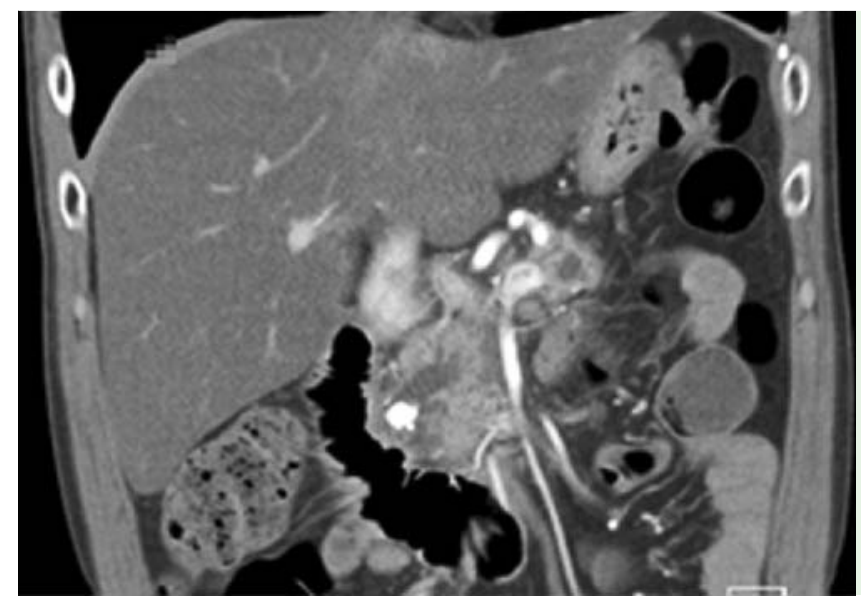

Fig. 1 A computed tomography scan at admission showing a pancreatic duct stone of $1.1 \mathrm{~cm}$ in diameter and pancreatic duct dilatation.

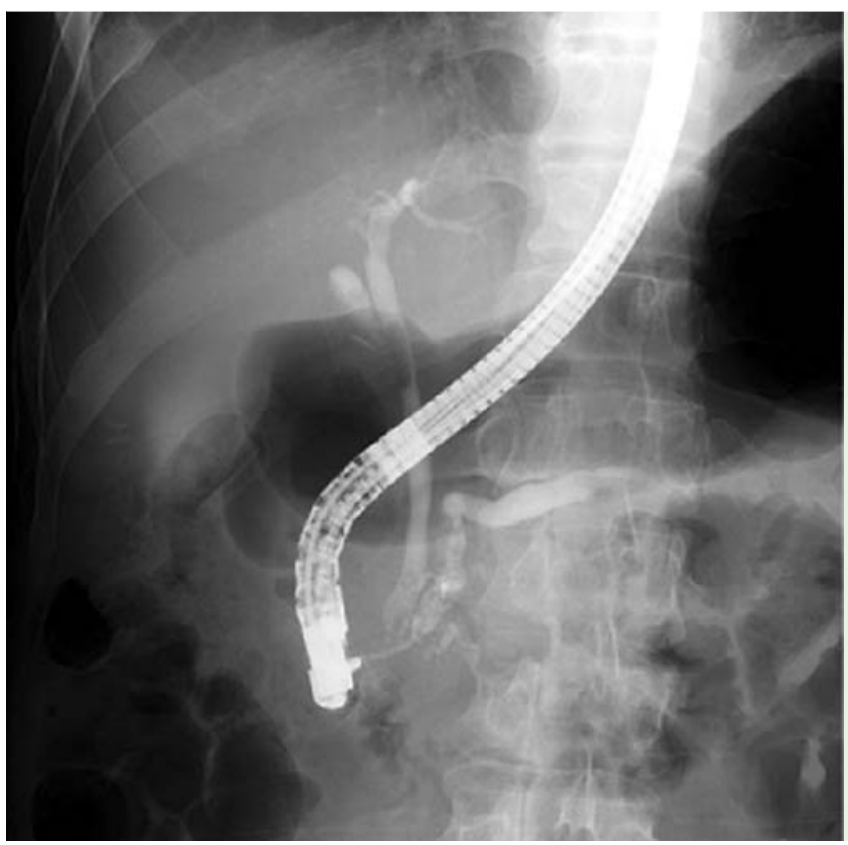

Fig. 2 At initial endoscopic retrograde cholangiopancreatography, the pancreatogram showed the stricture of the pancreatic duct downstream from the pancreatic duct stone.

A 51-year-old man with abdominal pain was admitted for treatment of alcoholrelated chronic pancreatitis with acute exacerbation. The acute episode was caused by a large impacted stone of $1.1 \mathrm{~cm}$ in diameter within the proximal main pancreatic duct ( Fig. 1 ). Stricture of the duct downstream from the stone was noted on initial endoscopic retrograde cholangiopancreatography (ERCP) ( $\bullet$ Fig. 2).

A stepwise approach was followed to remove the pancreatic duct stone. First, a short term, fully covered, self-expandable metal stent (SEMS; 10×40 mm, WallFlex; Boston Scientific, Natick, Massachusetts, USA) was placed in the pancreatic duct for 3 days to dilate the stricture ( $\bullet$ Fig. 3 ). Second, after radiographic confirmation of full stent expansion, the metal stent was removed and intraductal electrohydraulic lithotripsy (EHL; Lithotron EL-25 generator; Walz Electronic Inc., Rohrdorf, Germany) was performed to fragment the whitish stone ( $\bullet$ Fig. 4). EHL was performed under direct peroral pancreatoscopy (with carbon dioxide insufflation)

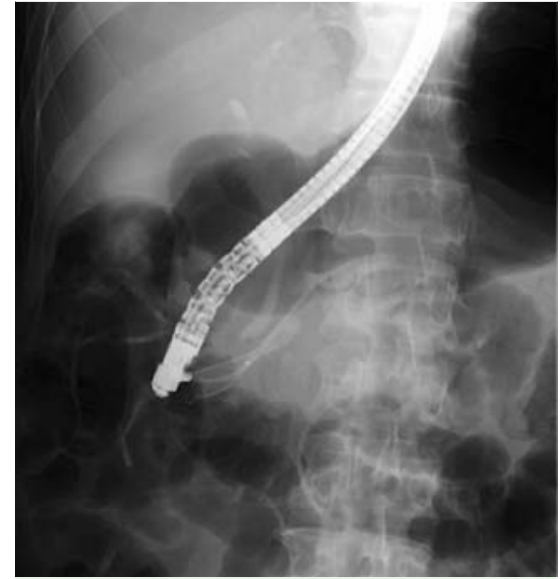

Fig. 3 Placement of a short term, fully covered, self-expandable metal stent $(10 \times 40 \mathrm{~mm})$ in the pancreatic duct to dilate the stricture.

using an ultraslim upper endoscope (GIFXP290N, outer diameter $5.4 \mathrm{~mm}$, working channel $2.2 \mathrm{~mm}$; Olympus, Tokyo, Japan). Finally, the fragmented stones were successfully removed using an ERCP basket ( Fig.5). No procedure-related complications occurred. The patient remained asymptomatic for 6 months with no recurrence of pancreatitis after the end of treatment.

Endoscopic extraction of large pancreatic duct stones is usually difficult, but is possible through the papilla if stone fragmentation is first performed by mechanical lithotripsy, extracorporeal shock wave lithotripsy, or intraductal EHL [1,2]. For intraductal EHL, pancreatoscopic visualization is necessary in order to avoid duct injury by the high energy of EHL [3]. In addition, dilation of a ductal stricture, if present, is required before proceeding to remove the stones. In the current case, short term placement of a fully covered SEMS was used for stricture dilation, and peroral pancreatoscopy with EHL using an ultraslim upper endoscope was performed successfully for fragmentation of the large pancreatic duct stone without complication.

Endoscopy_UCTN_Code_TTT_1AR_2AI

Competing interests: None 


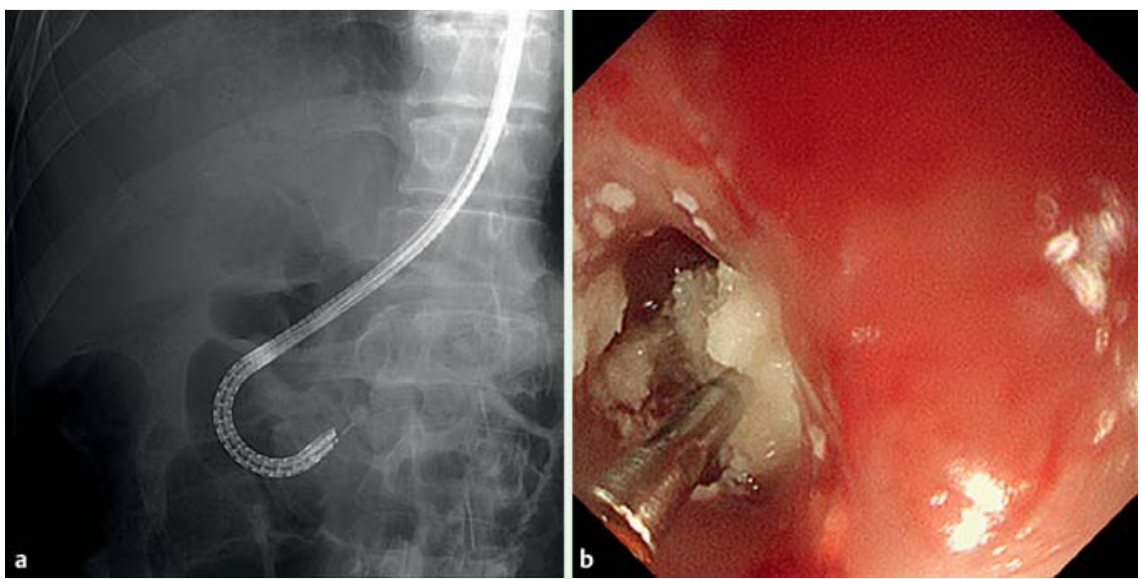

Fig.4 An embedded whitish stone was identified by peroral pancreatoscopy using an ultraslim upper endoscope. Electrohydraulic lithotripsy (EHL) was performed to fragment the stone. a Fluoroscopic image of stone fragmentation by EHL. b Endoscopic image of stone fragmentation by EHL.

\section{Seung Kak Shin, Jae Hee Cho, Yeon Suk Kim}

Department of Internal Medicine, Gachon University Gil Medical Center, Incheon, Republic of Korea

\section{References}

1 Delhaye M, Matos C, Deviere J. Endoscopic technique for the management of pancreatitis and its complications. Best Pract Res Clin Gastroenterol 2004; 18: 155-181

2 Farnbacher MJ, Schoen C, Rabenstein T et al. Pancreatic duct stones in chronic pancreatitis: criteria for treatment intensity and success. Gastrointest Endosc 2002; 56: $501-$ 506

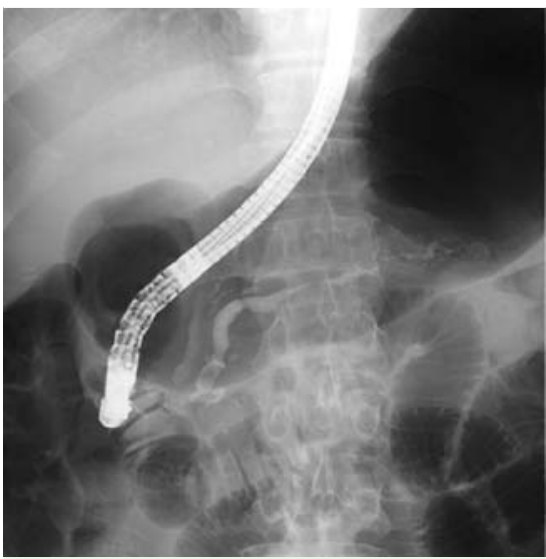

Fig. 5 Pancreatogram showing the complete removal of the pancreatic duct stone, with improvement of upstream duct dilatation and downstream duct stricture.

\section{Corresponding author}

\section{Jae Hee Cho, MD}

Department of Internal Medicine Gachon University Gil Medical Center

21 Namdone-daero

774 beon-gil

Namdong-gu

Incheon 405-760

Republic of Korea

Fax: +82-32-4603408

jhcho9328@gilhospital.com 